\title{
Metabolic cutis laxa syndromes
}

\author{
Miski Mohamed • Dorus Kouwenberg • \\ Thatjana Gardeitchik • Uwe Kornak • Ron A. Wevers • \\ Eva Morava
}

Received: 28 October 2010 /Revised: 11 February 2011 / Accepted: 17 February 2011 /Published online: 23 March 2011

(C) The Author(s) 2011. This article is published with open access at Springerlink.com

\begin{abstract}
Cutis laxa is a rare skin disorder characterized by wrinkled, redundant, inelastic and sagging skin due to defective synthesis of elastic fibers and other proteins of the extracellular matrix. Wrinkled, inelastic skin occurs in many cases as an acquired condition. Syndromic forms of cutis laxa, however, are caused by diverse genetic defects, mostly coding for structural extracellular matrix proteins. Surprisingly a number of metabolic disorders have been also found to be associated with inherited cutis laxa. Menkes disease was the first metabolic disease reported with old-looking, wrinkled skin. Cutis laxa has recently been found in patients with abnormal glycosylation. The discovery of the COG7 defect in patients with wrinkled, inelastic skin was the first genetic link with the Congenital
\end{abstract}

Communicated by: Jaak Jaeken

Competing interest: None declared.

M. Mohamed · D. Kouwenberg · T. Gardeitchik · E. Morava

Institute for Genetic and Metabolic Disease,

Radboud University Medical Centre Nijmegen,

P.O Box 9101, 6500 HB Nijmegen, The Netherlands

M. Mohamed · D. Kouwenberg $\cdot$ T. Gardeitchik $\cdot$ E. Morava $(\bowtie)$

Department of Pediatrics,

Radboud University Medical Centre Nijmegen,

P.O Box 9101, 6500 HB Nijmegen, The Netherlands

e-mail: E.Morava@cukz.umcn.nl

\section{R. A. Wevers}

Laboratory of Genetic, Endocrine and Metabolic Diseases,

Radboud University Medical Centre Nijmegen,

Nijmegen, The Netherlands

\section{U. Kornak}

Max Planck Institute for Molecular genetics,

Charité-Universitätsmedizin Berlin,

Berlin, Germany
Disorders of Glycosylation (CDG). Since then several inborn errors of metabolism with cutis laxa have been described with variable severity. These include P5CS, ATP6V0A2-CDG and PYCR1 defects. In spite of the evolving number of cutis laxa-related diseases a large part of the cases remain genetically unsolved. In metabolic cutis laxa syndromes the clinical and laboratory features might partially overlap, however there are some distinct, discriminative features. In this review on metabolic diseases causing cutis laxa we offer a practical approach for the differential diagnosis of metabolic cutis laxa syndromes.

\section{Introduction}

Cutis laxa (CL) is characterized by redundant, sagging, inelastic and wrinkling skin (Morava et al. 2009a; van Maldergem et al. 1989). This disorder of skin and connective tissue can be inherited (autosomal recessive, autosomal dominant and X-linked recessive) or acquired. Manifestations of $\mathrm{CL}$ occur generalized as well as local and might improve over time in some of the patients (Morava et al. 2006). Defective elastin synthesis and structural abnormalities in extracellular matrix proteins leading to CL can be further confirmed by demonstrating an abnormal histological staining and ultrastructure in skin biopsy (Urban et al. 2005).

Autosomal dominant cutis laxa (ADCL; MIM 123700) is a connective tissue disorder characterized by wrinkled and sagging inelastic skin associated with internal organ involvement (van Maldergem et al. 1989). Patients are usually diagnosed in early childhood with a mild form of cutis laxa with some systemic involvement (hernias, cardiovascular manifestations, gastrointestinal diverticuli and emphysema). ADCL is genetically heterogeneous: mutations have been 


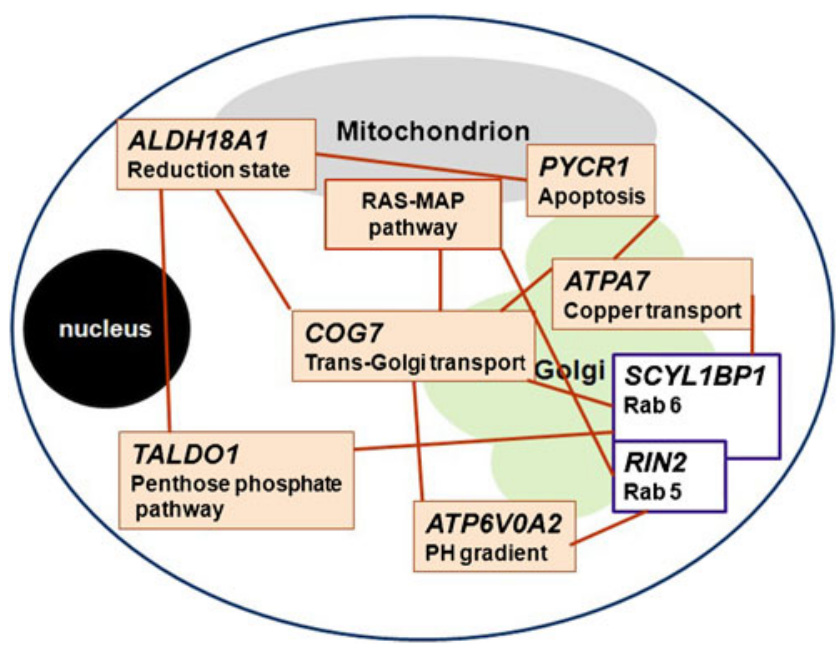

Fig. 1 Genetic defects related to biochemical pathways or inborn errors of metabolism in patients with cutis laxa and their suggested pathomechanism

reported in the elastin gene (ELN; MIM 130160) as well as in the fibulin-5 gene (FBLN5; MIM 604580) (Hu et al. 2006). Homozygous mutations in FBLN5 are associated with the more severe form of CL with extensive systemic involvement, (autosomal recessive cutis laxa type 1; ARCL1; MIM 219100).

ARCL1 is a distinct type of often lethal, generalized connective tissue disorder with severe systemic manifestations (Elahi et al. 2006). The skin manifestations affect the whole body and are usually recognizable from birth. Pulmonary emphysema develops in early childhood, often leading to respiratory failure. Vascular anomalies include arterial aneurysms causing progressive heart failure and genitourinary tract diverticuli leading to severe infections. Intellectual development appears to be within normal range (Mégarbané et al. 2009).

In the majority of cases with ARCL1 the genetic defect is not known. Mutations have been identified in the genes ELN,
FBLN5 and EFEMP2 (MIM 604633), encoding elastin, fibulin-5 and EGF-containing fibulin-like extracellular matrix protein 2 (Fibulin-4), respectively (Elahi et al. 2006; Hucthagowder et al. 2006; Mégarbané et al. 2009). Arachnodactyly, bone fractures and aneurysms are common in patients with EFEMP2 mutations (Hucthagowder et al. 2006). Severe respiratory involvement has been observed related to mutations in FBLN5 and LTBP4 (Elahi et al. 2006; Urban et al. 2009).

Autosomal recessive cutis laxa, type 2 (ARCL2A and ARCL2B; MIM 219200 and MIM 612940) is a more benign, genetically heterogeneous condition (Fig. 1), associated with cutis laxa with growth and developmental delay and skeletal anomalies (van Maldergem et al. 1989; Morava et al. 2005; Noordam et al. 2009). Patients with ARCL2 present with generalized cutis laxa at birth. Intellectual deficit and seizures have been reported in older patients (Morava et al. 2009a, b). The systemic manifestations are mild in ARCL2 and pulmonary emphysema and cardiac anomalies are rare. The underlying etiology in the majority of ARCL2 cases remains unknown; in some patients a combined disorder of N- and Olinked glycosylation (congenital disorder of glycosylation type II) has been detected due to mutations in the ATP6V0A2 gene (MIM 611716) (Morava et al. 2005; Kornak et al. 2008). There are only a few specific clinical features that distinguish ARCL2 patients with glycosylation defects from those without metabolic anomalies. Mutations in the PYCR1 gene (MIM 179035) (Fig. 2) have recently been identified in patients with ARCL2 with wrinkly skin, osteopenia and progeroid features, and the closely related de Barsy syndrome (DBS; MIM 219150) (Reversade et al. 2009). Mutations in another Golgi-related gene product, GORAB, have been found in several patients with gerodermia osteodysplastica (GO; MIM 231070), which can be regarded as a subform of ARCL2 (Hennies et al. 2008).

Inborn errors of metabolism such as Menkes syndrome (MIM 309400) are also associated with cutis laxa (Fig. 1).
Fig. 2 Metabolic pathways related to P5CS and PYCR1 defects

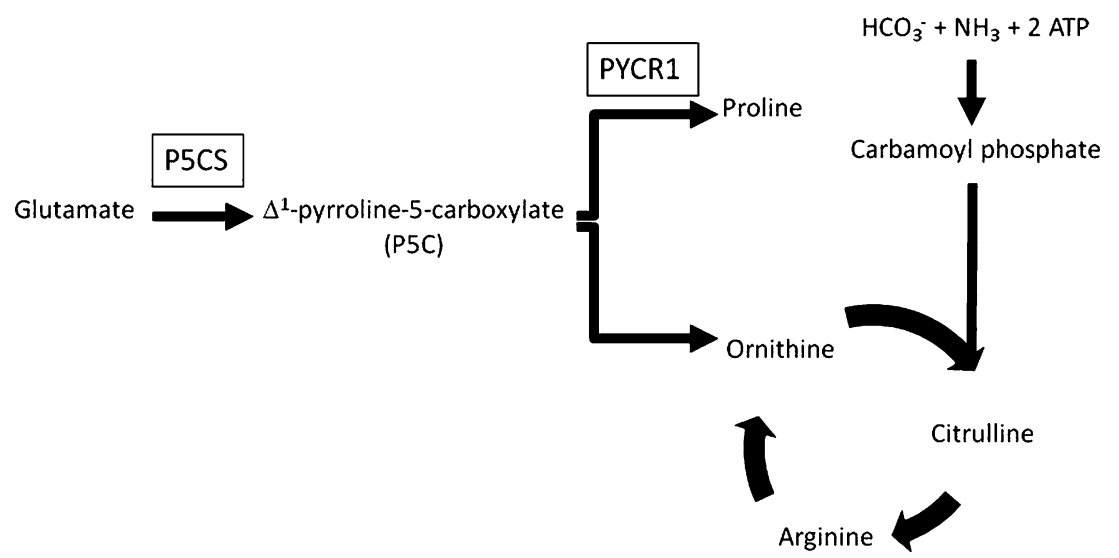


Fig. 3 Cutis laxa in a patient with ARCL2 demonstrating sagging truncal and abdominal skin (a), characteristic facial features and microcephaly in a patient with COG7-CDG (b), kinky, hypopigmented hair in X-linked cutis laxa syndrome (c) and downslanting palpebral fissures and sagging chin in patient with ATP6V0A2-CDG (d)
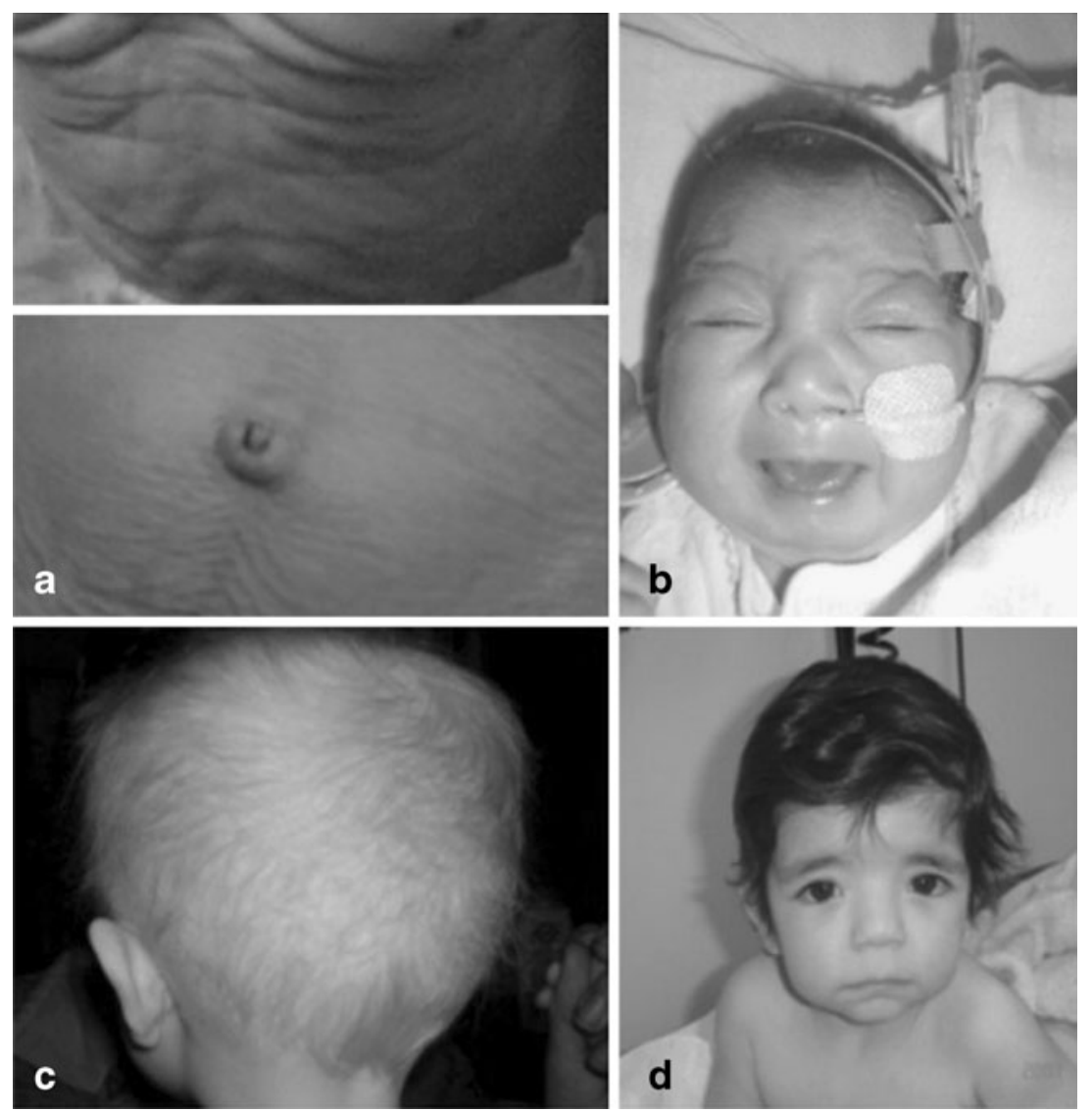

\section{Metabolic cutis laxa syndromes}

\section{Menkes disease}

In 1962 Menkes described an X-linked recessive disorder later to be known as Menkes disease (MD; MIM 309400), characterized by kinky hair, growth retardation and progressive neurological dysfunction with a fatal course. The characteristic abnormality of the scalp hair is caused by a combination of broken (trichorrhexis nodosa) and twisted (pili torti) hair of varying diameter of the hairshaft (Menkes et al. 1962) (Fig. 3c).

Subsequently, the classical phenotype was broadened by reporting hypothermia, skeletal anomalies and vascular defects due to a generalized connective tissue disorder in these patients. The physiopathology is entrapment of copper in the intestinal mucosa due to a defective transport mechanism, leading to systemic copper deficiency in multiple organs, especially in the brain (Danks et al. 1972).

MD is caused by a defect in the ATP7A gene (MIM 300011) leading to dysfunction of the ATP7A protein, a copper-transporting ATPase expressed in almost all tissues except the liver (Vulpe et al. 1993; Chelly et al. 1993; Mercer et al. 1993). Normally localized in the trans-Golgi network, it supplies the excreted copper-dependent enzymes with copper, facilitates the ATP fueled efflux in cells and the uptake of copper in the small intestine (Yamaguchi et al. 1996; Camakaris et al. 1995). In conditions where copper is increased it translocates to the plasma membrane and this copper efflux restores homeostasis (Petris et al. 1996). Dysfunction of this mechanism leads to decreased activity of the copper-dependent enzymes and deficiency of copper in the brain due to lack of transport across the blood-brain barrier. Failure of these copper-transport mechanisms has severe consequences. The classical Menkes phenotype leads to death in the first years. Since the initial description many other symptoms have been associated with MD (Table 1) and besides the classical, lethal phenotype milder forms have been described. Occipital Horn Syndrome (OHS MIM 304150 or X-linked cutis laxa), the mildest Menkes phenotype, manifests with cutis laxa (Fig. 3a), hernias, diverticula of the gastrointestinal tract and bladder, tortuosity of the arteries, hyperlaxity of the joints and skeletal anomalies. The neurological symptoms are less severe and life expectancy is longer than in classical Menkes disease. The most characteristic feature of OHS is the formation of occipital exostoses due to calcification of the attachments of the trapezius and sternocleidomastoid muscles to the occipital bone (Proud et al. 1996). 
Table 1 Clinical features in metabolic disorders associated with cutis laxa

\begin{tabular}{|c|c|c|c|c|c|c|}
\hline & ATP7 & COG7 & ATP6V0A2 & PYCR1 & P5CS & MAP-k pathway genes \\
\hline Cutis laxa & + & + & ++ & ++ & + & $+/-$ \\
\hline Psychomotor retardation & + & + & + & + & ++ & $+/-$ \\
\hline Seizures & ++ & + & $+/-$ & - & + & $+/-$ \\
\hline Neuropathy & - & + & - & - & + & - \\
\hline Hypotonia & - & ++ & + & - & ++ & + \\
\hline Hypertonia & + & - & - & - & - & - \\
\hline Athetoid movements & - & - & - & ++ & + & - \\
\hline Cobble stone-like brain defect & - & - & $++/-$ & - & - & - \\
\hline Corpus callosum hypoplasia/aplasia & - & - & - & $++/-$ & - & $+/-$ \\
\hline Open fontanel/late closure & - & - & ++ & - & - & - \\
\hline Microcephaly & + & ++ & + & $+/-$ & + & - \\
\hline Dysmorphic features & + & + & ++ & + & + & ++ \\
\hline Kinky, sparse hair & ++ & - & - & - & - & $+/-$ \\
\hline Hypopigmentation skin/nails & + & - & - & - & - & - \\
\hline Growth delay & ++ & ++ & + & + & + & + \\
\hline Hyperlaxity of joints & + & + & ++ & + & ++ & $+/-$ \\
\hline Adducted thumbs/distal arthrogryposis & - & ++ & - & ++ & - & - \\
\hline Spontaneous bone fracture & + & - & $+/-$ & $+/-$ & $+/-$ & - \\
\hline Hernias & ++ & - & + & ++ & - & $+/-$ \\
\hline VSD & - & + & - & - & - & $+/-$ \\
\hline Spontaneous vascular ruptures & + & - & - & - & - & - \\
\hline Hyperthermia & - & + & - & - & - & - \\
\hline Hypothermia & + & - & - & - & - & - \\
\hline Recurrent infections & - & + & - & - & - & - \\
\hline Cataracts & - & - & - & $+/-$ & ++ & - \\
\hline
\end{tabular}

To date more than 357 mutations (not all published) have been discovered in $\mathrm{MD}$ and $\mathrm{OHS}$ with great clinical heterogeneity, inter- and intrafamilial (Møller et al. 2009).

Diagnosis is based on clinical features, low serum copper and ceruloplasmin. Sequencing of the ATP7A gene is however the diagnostic gold standard. Unfortunately, treatment options are very limited and mostly symptomatic. Recent studies suggest that parenteral or subcutaneous copper administration could prolong survival in patients with partially active ATP7A function, when initiated soon after birth (Kaler et al. 2008).

\section{COG7-CDG}

Congenital disorders of glycosylation (CDG) form a heterogeneous group of inborn errors of metabolism characterized by a defective biosynthesis of glycans. These disorders are often multisystemic. The protein $\mathrm{N}$-glycosylation disorders can be subdivided in type I and II disorders based on the transferrin isoelectric focusing (TIEF) pattern. There are two main types of protein glycosylation of clinical significance: the N-glycosylation and the O-glycosylation. N-glycosylation starts in the cytoplasm and endoplasmatic reticulum (ER) where an oligosaccharide is assembled on a dolichol carrier and transferred to the nascent protein, which is then processed in the Golgi. O-glycosylation is limited to the Golgi. Defects in the N-glycosylation pathway generally show abnormal plasma TIEF profiles, whereas mucin type O-glycosylation defects lead to abnormal isofocusing of plasma apolipoprotein C-III (apoC-III) (Wopereis et al. 2006) .

Defects in the Conserved Oligomeric Golgi complex (COG) are mostly responsible for the recent expansion of novel CDG subtypes. COG7-CDG (CDG type IIe, MIM 608779 ) is caused by a mutation in subunit seven of the COG complex existing of eight subunits in total (Morava et al. 2007; $\mathrm{Ng}$ et al. 2007; Spaapen et al. 2005; Wu et al. 2004). The defect localized in the Golgi results in a combined $\mathrm{N}$ - and O-glycosylation defect.

To date six patients with COG7-CDG, all with a fatal outcome, have been described. All affected patients were from consanguineous descent. The clinical characteristics are growth retardation, microcephaly (Fig. 3b), hypotonia, cutis laxa or wrinkled skin, hepato-splenomegaly, skeletal dysplasia, cardiac anomalies, recurrent infections, feeding prob- 
lems, hyperthermia, adducted thumbs, mental retardation, seizures and structural brain abnormalities. Liver function is disturbed leading to elevated serum transaminases and bilirubin (Morava et al. 2007; Ng et al. 2007; Spaapen et al. 2005; Wu et al. 2004).

With the discovery of COG7-CDG, the first association between cutis laxa and glycosylation was discovered (Wu et al. 2004). So far no histological evaluation is available proving abnormal elastin structure underlying the skin anomalies in COG7-CDG patients. It is hypothesized that hypoglycosylation of extracellular matrix proteins, elastin and/or collagen, could be causative.

\section{ATP6V0A2-CDG}

Autosomal recessive cutis laxa type IIA (ARCL2A, Debré type MIM 219200) and wrinkly skin syndrome (WSS; MIM 278250) are characterized by excessive skin wrinkling or congenital cutis laxa, intrauterine growth retardation, failure to thrive, microcephaly, multiple dysmorphic features (Fig. 3d) and a large and late closing fontanel (Morava et al. 2005). Additionally, hypotonia, feeding problems, hyperlaxity of joints and an abnormal fat distribution might occur (Morava et al. 2008). The most common ocular anomalies are strabismus and myopia. Congenital hip dislocations and decreased bone mineral density are frequent skeletal findings. Developmental delay is observed in the majority of patients (mainly due to muscle hypotonia and joint laxity) but brain anomalies are rare. Neurological symptoms include psychomotor retardation, seizures, hearing loss and cobblestone like brain dysgenesis (Van Maldergem et al. 2008; Morava et al. 2009b). In this unique form of cutis laxa syndrome the skin features seem to improve over time.

The initial finding of a combined $\mathrm{N}$ - and O-linked glycosylation defect in children diagnosed with ARCL2 made the first link between CDG and extracellular matrix anomalies in cutis laxa patients (Morava et al. 2006). All affected patients showed a type 2 pattern on transferrin isoelectrofocusing (TIEF), abnormal isoelectrofocusing of apolipoprotein C-III (apoC-III) and abnormal mass spectrometry of glycans of total serum proteins (Morava et al. 2008; Kornak et al. 2008).

In the other group of children demonstrating wrinkled skin, a Golgi transport defect causing COG7-CDG, supplied further pathogenetic evidence for the role of glycosylation in the normal maturation of extracellular matrix proteins. Consequently, CDG screening was performed in a large group of consanguineous patients with ARCL2. Genetic analysis led to the discovery of different loss of function mutations in the vesicular $\mathrm{H}^{+}$-ATPase subunit a2, ATP6V0A2 (Kornak et al. 2008). Since then several different mutations have been detected in WSS and ARCL2 without a clear genotype-phenotype correlation (Hucthagowder et al.
2009). The mechanism, leading to cutis laxa in ATP6V0A2CDG patients has not yet been fully unraveled. Possible theories include an abnormal transport of extracellular matrix (ECM) proteins due to the v-ATPase related proton pump defect (Kornak et al. 2008; Guillard et al. 2009), delayed secretion of elastin and excessive storage of immature compounds like tropoelastin in the Golgi (Hucthagowder et al. 2009) and abnormal function and transport of glycosyltransferases leading to a hypoglycosylated state of diverse extracellular matrix components (Kornak et al. 2008; Hucthagowder et al. 2009).

\section{P5CS}

$\Delta^{1}$-pyrroline-5-carboxylate synthase (P5CS) (Fig. 2) catalyses the reduction of glutamate to $\Delta^{1}$-pyrroline-5-carboxylate (P5C), a precursor of proline and ornithine. Deficiency of P5CS leads to decreased levels of proline and ornithine in some patients. Proline, apart from its role in protein synthesis, is hypothesized to play a role in neurotransmission. Ornithine is an important intermediate in the urea cycle. Two isoforms of P5CS, generated by alternative splicing, are known: the short isoform mainly participates in arginine production in the gut whereas the long isoform is important for proline synthesis in various tissues (Phang et al. 1995; Valle and Simell 1995).

Deficiency of P5CS was first described in two Algerian siblings with progressive neurodegeneration, joint laxity, skin hyperlaxity and bilateral subcapsular cataracts.

A missense mutation, $\mathrm{R} 84 \mathrm{Q}$, altering a conserved residue in the P5CS $\gamma$-glutamyl kinase domain and thereby reducing the activity of both isoforms was found in the two siblings (Kamoun et al. 1998; Baumgartner et al. 2000).

The clinical phenotype in P5CS deficiency (MIM 138250) is characterized by cutis laxa, microcephaly, bilateral subcapsular cataract, severe mental retardation, joint laxity and hypotonia, structural brain abnormalities, progressive neurodegeneration, seizures, peripheral neuropathy and dystonic movements in hands and feet (Baumgartner et al. 2005). Biochemically, P5CS deficiency leads to a very typical pattern of paradoxical hyperammonemia as well as hypoprolinaemia and deficiency of urea cycle intermediates (ornithine, citrulline and arginine). Two pathophysiological explanations have been suggested for the clinical findings in P5CS deficiency. First, protein synthesis could be impaired in tissues depending highly on the endogenous production of proline, for example the production of collagen in connective tissues leading to cutis laxa (Smith and Phang 1978). But in this case rather an Ehlers-Danlos-like phenotype should be expected. The presence of a proline transporter in synaptic vesicles and axon terminals of glutamatergic neurons suggest a role of proline in neurotransmission. This might explain the severe mental retardation in the patients (Shafqat et al. 1995). 
Alternatively, proline has been suggested to play a role in protecting cells from oxidative stress (Phang et al. 2008). Errors in proline synthesis could therefore lead to developmental defects through increased apoptosis. Low levels of ornithine impair the urea cycle and thus lead to low serum levels of citrulline and arginine, and hyperammonemia. Following a meal, dietary arginine will temporarily restore the urea cycle, explaining the paradoxical character of the hyperammonemia.

Bicknell et al. described a family with four members affected by autosomal recessive cutis laxa due to a C-terminal P5CS missense mutation (Bicknell et al. 2008). Detailed biochemical serum analysis only revealed mildly low ornithine levels under fasting conditions, but no significantly altered proline concentrations. Furthermore, the rate of proline biosynthesis from glutamate was normal in patient fibroblasts. In contrast, the clinical presentation was highly similar to the previously reported cases casting doubt on the role of proline synthesis itself in the disease mechanism.

Only a few patients have been reported, and evidence-based treatment options are thus very limited. Trials with ornithine treatment showed poor results (Baumgartner et al. 2005).

\section{PYCR1}

Pyrroline-5-carboxylate reductase 1 (PYCR1) catalyses the reduction of $\Delta^{1}$-pyrroline-5-carboxylate (P5C) to proline, the final step in the synthesis of proline from glutamate (Fig. 2) (Adams and Frank 1980). Like P5CS, it plays a role in the biosynthesis of proline. PYCR1 is most highly expressed in bone and skin and is localized in mitochondria (Fig. 1).

Clinical features in PYCR1 deficient individuals (MIM 179035) include cutis laxa (most pronounced on the dorsum of hands and feet), dysmorphic features, osteopenia, distal arthrogryposis, joint laxity, hernias, mild mental retardation, athetoid movements and aplasia or hypoplasia of the corpus callosum. Although hypoprolinaemia would be expected, based on PYCR1 function, serum proline levels in PYCR1 deficient patients were within the normal range (Reversade et al. 2009). In urine, low-normal levels were found.

Guernsey et al. (2009) described a homozygous mutation in the PYCR1 gene, localized on chromosome 17, in five members of two Canadian families. This missense mutation, R266Q, leads to a premature termination of the PYCR1 gene product leading to loss of function. Eight additional mutations have been found in 35 members of 22 families. PYCR1 mutations have been found in various cutis laxa syndrome phenotypes, including De Barsy syndrome, Wrinkly Skin syndrome and also in ARCL2 patients, showing a similar clinical presentation as those with ATP6VOA2 defect (Leao-Teles et al. 2010).

Upon oxidative stress PYCR1-deficient fibroblasts showed low mitochondrial membrane potential, a disrup- tion of the mitochondrial network, and increased apoptosis rates. Likewise, knockdown of PYCR1 in Xenopus and zebrafish entailed epidermal hypoplasia and blistering, accompanied by a massive increase in apoptosis (Reversade et al. 2009). Addition of exogenous proline did not influence these effects observed in vitro and in vivo.

Although P5CS and PYCR1 defects are located in the same pathway, patients with PYCR1 mutations are less severely affected and have a better prognosis due to the absence of neurological involvement. This can be explained by the ubiquitous presence of a highly identical paralogue, PYCR2. P5CS is only encoded by a single gene.

As discussed for P5CS, it is not sure that proline synthesis plays the main role in the disease process. No treatment is available.

\section{RIN2}

Basel-Vanagaite et al. recently described a new elastic tissue disorder in three patients from two related consanguineous Israeli-Arab families (Basel-Vanagaite et al. 2009). Clinical features included soft, redundant facial skin, macrocephaly, downslanting palpebral fissures, puffy eyelids, sagging cheeks, everted lower lip, micrognathia and abnormal skull morphology, gingival hypertrophy, irregular dentition, sparse scalp hair and severe joint hyperlaxity and scoliosis. Psychomotor development was normal. The syndrome was termed MACS: macrocephaly, alopecia, cutis laxa and scoliosis (MIM 613075). Genetic analysis revealed a single base pair deletion in the RIN2 gene on chromosome 20. Afterwards a very similar phenotype was described in three siblings from a consanguineous Algerian family (Syx et al. 2010). A deletion in the RIN2 gene was found in these patients.

The gene product, the Ras and Rab interactor 2 protein, has been proven to interact with the Rab5 protein, which is required for endosomal trafficking (Saito et al. 2002). Endosomal trafficking was not impaired in RIN2-deficient patients, but it has been suggested that RIN2-deficiency (MIM 610222) is responsible for the phenotype through impairment of other trafficking pathways, eg ER-to-Golgi or Golgi-to-plasma membrane (Syx et al. 2010). Fibroblasts of the patients reported so far showed abnormal morphology of the Golgi apparatus with swollen cisternae and vacuole accumulation. Biochemical investigations in one of the patients showed normal plasma $\mathrm{N}$-glycans and variable alterations of the mucin type $O$-glycan Apolipoprotein $\mathrm{C}_{\text {- }} \mathrm{III}_{1}$, similar to the profile seen in patients with abnormal Golgi-related O-glycosylation (Albrecht et al. 2010; Wopereis et al. 2003, 2006). Besides their role for endosome function RIN proteins have also been described as a regulator of the Raspathway. This function could explain the weak overlap 
between MACS syndrome and Costello syndrome (sparse hair, prominent lips, cutis laxa) (see below).

Metabolic pathway defects described in association with wrinkled skin or cutis laxa

Transaldolase defiency, caused by a mutation in the TALDO1 gene (MIM 602063), presents with aortic coarctation, bleeding tendency, hepatosplenomegaly, telangiectasias of the skin and enlarged clitoris. Biochemical investigations showed thrombocytopenia and elevated concentrations of ribitol, D-arabitol and erythritol in urine and plasma. Recently erythronic acid was described as a novel biomarker for this disease (Engelke et al. 2010). One of the patients with this rare condition also showed severe cutis laxa phenotype. So far, there is no evidence, whether the association of TALDO1 defect and cutis laxa is a coincidence (Valayannopoulos et al. 2006).

Heterozygous germline mutations in genes encoding enzymes and regualators of the Ras-MAPK pathway cause Noonan syndrome (MIM 163950) and the related disorders Cardio-Facio-Cutaneous (MIM 115150), LEOPARD (MIM 151100 and 611554) and Costello syndrome (MIM 218040) (Gelb and Tartaglia 2006). These are clinically characterized by distinctive facial appearance, heart defects, ectodermal abnormalities, variable cognitive defects, motor delay and susceptibility to certain malignancies. Mutations in several genes (PTPN11, RAF, NF, HRAS, BRAF, MEK1/2, KRAS, SOS, SHOC2, NRAS and RAFl) have been identified in patients, all regulating the Ras-MAPK cascade (Sarkozy et al. 2009). Several patients have been reported with wrinkled skin and cutis laxa, especially in the inguinal, axillary regions, and affecting the hands and feet, caused by diverse mutations associated with Noonan, Cardio-Facio-Cutaneous or Costello syndromes (Kleefstra et al. 2011).
Gerodermia osteodysplastica (MIM 231070) is a clinical syndrome first described in 1950 by Bamatter et al. (Bamatter et al. 1950). It is characterized by lax, wrinkled skin, joint hyperlaxity, progeroid facial appearance, severe osteoporosis, malar and mandibular hypoplasia and growth retardation. The disorder is also known as 'Walt Disney dwarfism' due to the typical facial features (Bamatter et al. 1950). In a recent study by Hennies et al., genetic analysis of affected individuals from 13 families revealed nine pathogenic mutations in the GORAB (SCYL1BPI) gene (Hennies et al. 2008). The gene product GORAB interacts with Rab6, a protein involved in intracellular trafficking (Grigoriev et al. 2007). Interestingly, Rab6 has been demonstrated to interact with the conserved oligomeric Golgi complex (COG) (Sun et al. 2007). However, glycosylation defects have not been observed in GORAB deficiency (MIM 607983) (Hennies et al. 2008).

\section{Discussion}

Cutis laxa and wrinkled skin are frequently associated with diverse metabolic conditions. The majority of inborn errors presenting with cutis laxa are related to abnormal Golgi function and frequently related to transport defects and abnormal protein glycosylation. Most of these syndromes have both characteristic biochemical and clinical markers facilitating timely diagnosis. Patients with X-linked cutis laxa share the facial features and late closure of the fontanel with ATP6VOA2-related ARCL2, however they also present with the pathognomic radiological features of frontal horns, as well as abnormal hair growth and severe muscle involvement. In ATP6V0A2, failure to thrive, transient feeding problems and the eventual presence of cobble-stone like brain malformations are helpful in the diagnosis.

Table 2 Biochemical features in metabolic disorders associated with cutis laxa

\begin{tabular}{|c|c|c|c|c|c|c|}
\hline & ATP6V0A2 & COG7 & ATP7 & PYCR1 & $\mathrm{P} 5 \mathrm{CS}$ & MAP-k pathway genes \\
\hline Abnormal TIEF & + & + & - & - & - & - \\
\hline Abnormal ApoC-III IEF & + & + & - & - & - & - \\
\hline Decreased serum copper & - & - & + & - & - & - \\
\hline Decreased serum ceruloplasmin & - & - & + & - & - & - \\
\hline Elevated serum lactic acid & $+/-$ & $+/-$ & - & $+/-$ & - & $+/-$ \\
\hline Elevated serum ammonia & - & - & - & - & $+(-)$ & - \\
\hline Decreased serum proline & - & - & - & - & $+(-)$ & - \\
\hline Decreased serum citrulline & - & - & - & - & $+(-)$ & - \\
\hline Other aminoacids & - & - & - & - & - & Increased alanine $+/-$ \\
\hline Endocrine abnormailties & $+/-$ & - & - & - & - & - \\
\hline Liver dysfunction & $+/-$ & ++ & - & - & - & - \\
\hline Elevated serum creatine kinase & - & ++ & - & - & - & $+/-$ \\
\hline
\end{tabular}


Patients with COG7-CDG have heart malformations, a less severe skin involvement and more severe microcephaly. Disorders of the MAP kinase pathway have distinct dysmorphic features, short stature and other ectodermal anomalies, like slow hair and nail growth. This is in many ways similar to the ectodermal features of patients with a RIN2 defect. PYCR1 mutation carriers have characteristic features as well, including neonatal progeria, corpus callosum agenesis, arthrogryposis and abnormal movements. These symptoms are mostly absent in other types of cutis laxa.

COG7-CDG and ATP6V0A2-CDG are combined N- and O-linked glycosylation disorders (patients are demonstrating an abnormal TIEF and apo-CIII IEF). Menkes disease and X-linked cutis laxa have abnormal copper metabolism leading to easily detectable abnormalities in serum and urine but normal TIEF and apoC-III IEF. Patients with RIN2 defect have also normal serum TIEF pattern but have been associated with abnormal O-glycosylation (Albrecht et al. 2010).

Contrary to patients with Golgi system-related cutis laxa forms, those with PYCR1 and MAP kinase pathway defects show no glycosylation abnormalities. In some of these children mild mitochondrial dysfunction has been observed, but usually they do not manifest metabolic markers of mitochondrial disease.

The clinical finding of wrinkled skin or cutis laxa appears to have a growing significance due to its occurrence in several inborn errors. Although the pathogenetic explanation is not completely understood in many cases, we suggest that the finding of wrinkled skin in a child with developmental delay should evoke detailed metabolic evaluation. The initial step is to perform a skin biopsy for histology and electron microscopy in order to differentiate between collagen and elastin abnormalities. The combination of elastin fragmentation in the skin biopsy and abnormalities of the TIEF and/or Apo C-III IEF might lead directly to the diagnosis of ATP6V0A2-CDG. Less severe elastin abnormalities are suggestive for COG7-CDG. Copper and ceruloplasmin are reliable screening tools when X-linked cutis laxa or Menkes Disease are suspected. Routine metabolic screening for ammonia, lactic acid and serum amino acid levels can give clues about the possible underlying metabolic disease, especially in P5CS, PYCR1 or MAP kinase defects (Table 2). More specific laboratory investigations or direct mutation analysis can be performed based on pathognomic clinical features and discriminative biochemical results (Tables 1 and 2).

Open Access This article is distributed under the terms of the Creative Commons Attribution Noncommercial License which permits any noncommercial use, distribution, and reproduction in any medium, provided the original author(s) and source are credited.

\section{References}

Adams E, Frank L (1980) Metabolism of proline and the hydroxyprolines. Annu Rev Biochem 49:1005-1061

Albrecht B, De Brouwer AP, Lefeber DJ (2010) MACS syndrome: a combined collagen and elastin disorder due to abnormal golgi trafficking. Am J Med Genet A; in press

Bamatter F, Franceschetti A, Klein D, Sierro A (1950) Gerodermie osteodysplastique hereditaire. Ann Pédiatr 174:126-127

Basel-Vanagaite L, Sarig O, Hershkovitz D et al (2009) RIN2 deficiency results in macrocephaly, alopecia, cutis laxa, and scoliosis: MACS syndrome. Am J Hum Genet 85:254-263

Baumgartner MR, Hu C-A, Almashanu S et al (2000) Hyperammonemia with reduced ornithine, citrulline, arginine and proline: a new inborn error caused by a mutation in the gene encoding $\Delta^{1}$-pyrroline-5-carboxylate synthase. Hum Mol Genet 9(19):2853-2858

Baumgartner MR, Rabier D, Nassogne M-C (2005) $\Delta^{1}$-pyrroline-5carboxylate synthase deficiency: neurodegeneration, cataracts and connective tissue manifestations combined with hyperammonaemia and reduced ornithine, citrulline, arginine and proline. Eur $\mathrm{J}$ Pediatr 164:31-36

Bicknell LS, Pitt J, Aftimos S, Ramadas R, Maw MA, Robertson SP (2008) A missense mutation in ALDH18A1, encoding $\Delta^{1}$ pyrroline-5-carboxylate synthase (P5CS), causes an autosomal recessive neurocutaneous syndrome. Eur J Hum Genet 16:11761186

Camakaris J, Petris MJ, Bailey L et al (1995) Gene amplification of the Menkes (MNK;ATP7A) P-type ATPase gene of CHO cells is associated with copper resistance and enhanced copper efflux. Hum Mol Genet 4:2117-2123

Chelly J, Tümer Z, Tønnesen T et al (1993) Isolation of a candidate gene for Menkes disease that encodes a potential heavy metal binding protein. Nat Genet 3:14-19

Danks DM, Campbell PE, Stevens BJ, Mayne V, Cartwright E (1972) Menke's kinky hair syndrome: an inherited defect in copper absorption with widespread effects. Pediatrics 50:188-201

Elahi E, Kalhor R, Banihosseini SS et al (2006) Homozygous missense mutation in fibulin-5 in an Iranian autosomal recessive cutis laxa pedigree and associated haplotype. J Invest Dermatol 126:1506-1509

Engelke UF, Zijlstra FS, Mochel F et al (2010) Mitochondrial involvement and erythronic acid as a novel biomarker in transaldolase deficiency. Biochim Biophys Acta 1802:1028-1035

Gelb BD, Tartaglia M (2006) Noonan syndrome and related disorders: dysregulated RAS-mitogen activated protein kinase signal transduction. Hum Mol Genet 15(2):R220-R226

Grigoriev I, Splinter D, Keijzer N et al (2007) Rab6 regulates transport and targeting of exocytotic carriers. Dev Cell 13:305-314

Guernsey DL, Jiang H, Evans SC et al (2009) Mutation in pyrroline-5carboxylate reductase 1 gene in families with cutis laxa type 2 . Am J Hum Genet 85:120-129

Guillard M, Dimopoulou A, Fischer B et al (2009) Vacuolar H+-ATPase meets glycosylation in patients with cutis laxa. Biochim Biophys Acta 1792:903-914

Hennies HC, Kornak U, Zhang H et al (2008) Gerodermia osteodysplastica is caused by mutations in SCYL1BP1, a Rab-6 interacting golgin. Nat Genet 40:1410-1412

Hu Q, Loeys BL, Coucke PJ et al (2006) Fibulin-5 mutations: mechanisms of impaired elastic fiber formation in recessive cutis laxa. Hum Mol Genet 15:3379-3386

Hucthagowder V, Sausgruber N, Kim KH et al (2006) Fibulin-4: a novel gene for an autosomal recessive cutis laxa syndrome. Am J Hum Genet 78:1075-1080 
Hucthagowder V, Morava E, Kornak U, Lefeber DJ et al (2009) Loss-of-function mutations in ATP6V0A2 impair vesicular trafficking, tropoelastin secretion and cell survival. Hum Mol Genet 18:2149-2165

Kaler SG, Holmes CS, Goldstein DS et al (2008) Neonatal diagnosis and treatment of Menkes disease. N Engl J Med 358:605-614

Kamoun P, Aral B, Saudubray JM (1998) A new inherited metabolic disease: delta1-pyrroline 5-carboxylate synthetase deficiency. Bull Acad Natl Méd 182(1):131-137, discussion 138-139

Kleefstra T, Wortmann SB, Rodenburg RJ, Bongers EM et al (2011) Mitochondrial dysfunction and organic aciduria in five patients carrying mutations in the Ras-MAPK pathway. Eur J Hum Genet $19: 138-144$

Kornak U, Reynders E, Dimopoulou A et al (2008) Impaired glycosylation and cutis laxa caused by mutations in the vesicular ${ }^{\mathrm{H}+}$-ATPase subunit ATP6V0A2. Nat Genet 40:32-34

Leao-Teles E, Quelhas D, Vilarinho L, Jaeken J (2010) De Barsy syndrome and ATP6V0A2-CDG. Eur J Hum Genet 18:526

Mégarbané H, Florence J, Oliver Sass J et al (2009) An autosomalrecessive form of cutis laxa is due to homozygous elastin mutations, and the phenotype may be modified by a heterozygous fibulin 5 polymorphism. J Invest Dermatol 129:1650-1655

Menkes JH, Alter M, Steigleder GK, Weakley DR, Sung JH (1962) A sex-linked recessive disorder with retardation of growth, peculiar hair, and focal cerebral and cerebellar degeneration. Pediatrics 29:764-779

Mercer JF, Livingston J, Hall B et al (1993) Isolation of a partial candidate gene for Menkes disease by positional cloning. Nat Genet 3:20-25

Møller LB, Mogensen M, Horn N (2009) Molecular diagnosis of Menkes disease: genotype-phenotype correlation. Biochimie 91:1273-1277

Morava E, Wopereis S, Coucke P et al (2005) Defective protein glycosylation in patients with cutis laxa syndrome. Eur J Hum Genet 13:414-421

Morava E, Willemsen MA, Wopereis S, Ter Laak H, Lefeber DJ, Wevers RA, Cruysberg JR (2006) High myopia and congenital myopathy with partial pachygyria in cutis laxa syndrome. Eur J Ophthalmol 16:190-194

Morava E, Zeevaert R, Korsch E et al (2007) A common mutation in the COG7 gene with a consistent phenotype including microcephaly, adducted thumbs, growth retardation, VSD and episodes of hyperthermia. Eur J Hum Genet 15:638-645

Morava E, Lefeber DJ, Urban Z et al (2008) Defining the phenotype in an autosomal recessive cutis laxa syndrome with a combined congenital defect of glycosylation. Eur J Hum Genet 16:28-35

Morava E, Guillard M, Lefeber DJ, Wevers RA (2009a) Autosomal recessive cutis laxa syndrome revisited. Eur J Hum Genet 17:10991110

Morava E, Lefeber DJ, Wevers RA, Willemsen MA (2009b) Cobbelstone-like brain dysgenesis in cutis laxa syndrome. Neurology 73:1164

Ng BG, Kranz C, Hagebeuk EEO et al (2007) Molecular and clinical characterization of a Moroccan $\operatorname{Cog} 7$ deficient patient. Mol Genet Metab 91:201-204

Noordam C, Funke S, Knoers NV, Jira P, Wevers RA, Urban Z, Morava E (2009) Decreased bone density and treatment in patients with autosomal recessive cutis laxa. Acta Paediatr 98:490-494

Petris MJ, Mercer JF, Culvenor JG, Lockhart P, Gleeson PA, Camakaris J (1996) Ligand regulated transport of the Menkes copper P-type ATPase efflux pump from the Golgi apparatus to the plasma membrane: a novel mechanism of regulated trafficking. EMBO J 15:6084-6095
Phang JM, Yeh GC, Scriver CR (1995) Disorders of proline and hydroxyproline metabolism. In: Scriver CR, Beaudet AL, Sly WS, Valle D (eds) The Metabolic and Molecular Bases of Inherited Disease. McGraw Hill, New York, pp 1125-1146

Phang JM, Pandhare J, Liu Y (2008) The metabolism of proline as microenvironmental stress substrate. J Nutr 138:2008S-2015S

Proud VK, Mussell HG, Kaler SG, Young DW, Percy AK (1996) Distinctive Menkes disease variant with occipital horns: delineation of natural history and clinical phenotype. Am J Med Genet 65:44-51

Reversade B, Escande-Beillard N, Dimopoulou A et al (2009) Mutations in PYCR1 cause cutis laxa with progeroid features. Nat Genet 41:1016-1021

Saito K, Murai J, Kajiho H, Kontani K, Kurosu H, Katada T (2002) A novel binding protein composed of homophilic tetramer exhibits unique properties for the small GTPase Rab5. J Biol Chem 277:3412-3418

Sarkozy A, Carta C, Moretti S et al (2009) Germline BRAF mutations in Noonan, LEOPARD, and cardiofaciocutaneous syndromes: molecular diversity and associated phenotypic spectrum. Hum Mutat 30:695-702

Shafqat S, Velaz-Faircloth M, Henzi VA et al (1995) Human brainspecific L-proline transporter: molecular cloning functional expression and chromosomal localization of the gene in human and mouse genomes. Mol Pharmacol 48:219-229

Smith RJ, Phang JM (1978) Proline metabolism in cartilage: the importance of proline biosynthesis. Metabolism 27:685

Spaapen LJ, Bakker JA, van der Meer SB et al (2005) Clinical and biochemical presentation of siblings with COG-7 deficiency, a lethal multiple O- and N-glycosylation disorder. J Inherit Metab Dis 28:707-714

Sun Y, Shestakova A, Hunt L, Sehgal S, Lupashin V, Storrie B (2007) Rab6 regulates both ZW10/RINT-1 and conserved oligomeric Golgi complex-dependent Golgi trafficking and homeostasis. Mol Biol Cell 18:4129-4142

Syx D, Malfait F, Van Laer L (2010) The RIN2 syndrome: a new autosomal recessive connective tissue disorder caused by deficiency of Ras and Rab interactor 2 (RIN2). Hum Genet 128:79-88

Urban Z, Gao J, Pope FM, Davis EC (2005) Autosomal dominant cutis laxa with severe lung disease: synthesis and matrix deposition of mutant tropoelastin. J Invest Dermatol 124:1193-1199

Urban Z, Hucthagowder V, Schürmann N et al (2009) Mutations in LTBP4 cause a syndrome of impaired pulmonary, gastrointestinal, genitourinary, musculoskeletal, and dermal development. Am J Hum Genet 85:593-605

Valayannopoulos V, Verhoeven NM, Mention K et al (2006) Transaldolase deficiency: a new cause of hydrops fetalis and neonatal multi-organ disease. J Pediatr 149:713-717

Valle D, Simell O (1995) The hyperornithemias. In: Scriver CR, Beaudet, Sly WS, Valle D (eds) The Metabolic and Molecular Bases of Inherited Disease. McGraw Hill, New York, pp 1147 1185

Van Maldergem L, Ogŭr G, Yüksel M et al (1989) Facial anomalies in congenital cutis laxa with retarded growth and skeletal dysplasia. Am J Med Genet 32:265

Van Maldergem L, Yuksel-Apak M, Kayserili H et al (2008) Cobblestone-like brain dysgenesis and altered glycosylation in congenital cutis laxa, Debre type. Neurology 71:16021608

Vulpe C, Levinson B, Whitney S, Packman S, Gitschier J (1993) Isolation of a candidate gene for Menkes disease and evidence that it encodes a copper-transporting ATPase. Nat Genet $3: 7-13$ 
Wopereis S, Grünewald S, Morava E et al (2003) Apolipoprotein C-III isofocusing in the diagnosis of genetic defects in O-glycan biosynthesis. Clin Chem 49:1839-1845

Wopereis S, Lefeber DJ, Morava E, Wevers RA (2006) Mechanisms in protein O-glycan biosynthesis and clinical and molecular aspects of protein O-glycan biosynthesis defects: a review. Clin Chem 52:574-600
Wu X, Steet RA, Bohorov O et al (2004) Mutation of the COG complex subunit gene COG7 causes a lethal congenital disorder. Nat Med 10:518-523

Yamaguchi Y, Heiny ME, Suzuki M, Gitlin JD (1996) Biochemical characterization and intracellular localization of the Menkes disease protein. Proc Natl Acad Sci USA 93(24):1403014035 